\title{
Contents Vol. 92, 1936
}

\section{-MONATSSCHRIET \\ FOR \\ PSYCHIATRIE UND NEUROLOGIE}

\author{
BEGRUNDET VON C. WERNICKE UND TH. ZIEHEN \\ UNTER MITWIRKUNG VON \\ E. GAMPER \\ K. KLEIST HANSW.MAIER \\ P.SCHRODER \\ Frankfurt a. M. Zurich Wien \\ 0. POTZL \\ Prag \\ Frankfurt a. M. \\ HERAUSGEGEBEN VON \\ K. BOtfHOEFFEIl \\ BERLIN
}

Bd. 92

Mit i Tafel und zahlreichen Abbildungen im Text

\section{S.ißK.}

B E R LIN 1936 
Alle Rechte vorbehalten.

CTgW. g. Druok von B.. Wagner Sohn in Weimar. Printed in Germany. 


\section{Inhaltsverzeichnis.}

Originalarbeiten.

v. Bagothay, Ladislaus, Beitrag zu den Entstehungsbedingungen des pendelnden Kniereflexes im Zusammenhang mit einem Falle von multipler Sklerose

40

Beck, Ed., Zwangslaufige antagonistische Innervation bei einem Fall mit progressiver Versteifung, epileptischen und halluzinatorischen Anfallen

I

Becker, Erika, Klinische und anatomische Beitrage zur Pickschen Krankheit.

Cosack, Herta, Kasuistischer Beitrag zur Pathogenese der

Alkoholpsychosen.

Grotjahn, M., Zur Klinik und Psychologie der juvenilen

Paralyse

Haselager, Ph. J., Symptomatische Psychose bei der Recklinghausenschen Krankheit

Klein, R., Beitrag $\quad$ zur Frage der hemianopischen Hallu-

zinationen

Meyer, Fr., Mit Gesichtshauthamangiom vergesellschaftetes

Gehirnrankenangiom

Mutter, Reinhold F. G., Altindische Psychiatrie

Munzer, Franz Th., Das Kopfbeugungs-Symptom (Kb.-S.). Ein

differential-diagnostisches Zeichen bei Affektionen der hinteren

Ruckenmarks-Wurzeln. (Hierzu Taf. I) . 202

Pisk, Gerhart, Zur Frage der Pseudohalluzinationen bei der

Schizophrenie und ihrer Beziehungen zur eidetischen

Anlage

Polzfufi, Martin, Experimentelle und klinische Untersuchungen uber die Wirksamkeit des Cholereticums Curcumen

bei Nervenleiden

Potzl, 0. und H. Urban, tJber die isoliert erhaltene temporale Sichel bei zerebraler Hemianopsie.

67

Roggenbau, Christel, Zur Frage der korperlichen Storungen der endogenen Psychosen und ihrer Verwertbarkeit in differentialdiagnostischer Hinsicht 
Silberpfennig, Ida, Die Klinik der medialen Akustikus-

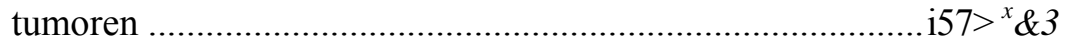

Stief, A., Beitrage zur Frage der endolumbalen Hormon-

behandlungen. (tlber die Wirkung von endolumbal

verabreichtem Insulin und Glanduitrin bei Schizo-

phxenie) ' .......................... 346

Stransky, Erwin, Zum Schizoidproblem...................................... 316

Szeky, Anton, Knochen- und Gelenksveranderungen der metaluetischen Nervenkrankheiten

45

Urban, $H$. siehe Potzl, 0 .

Urban, H., Zur Physiologie der Okzipitalregion des Menschen 32

Wenderowic, Eugen, "Uber die Einwirkungsart des Schlafenund des Stirnlappens auf das Kleinhirn

56 